\title{
Ultrasound-Assisted Extraction for Simultaneous Quantitation of Potential Sweetening Compounds from Derris reticulata Aqueous Extracts: A Response Surface Methodology Approach
}

\section{Keerati Thamapan}

Division of Biochemical Technology, School of Bioresources and Technology

Natta Laohakunjit ( $\square$ nutta.lao@kmutt.ac.th )

Division of Biochemical Technology, School of Bioresources and Technology https://orcid.org/00000002-2833-6273

\section{Orapin Kerdchuchen}

Division of Biochemical Technology, School of Bioresources and Technology

\section{Punchira Vongsawasdi}

Department of Microbiology, Faculty of Science

\section{Withawat Mingvanish}

Department of Chemistry, Faculty of Science

\section{Research}

Keywords: ultrasound-assisted extraction, phenolics, flavonoids, 18ß-glycyrrhetinic acid, sweet Thai plant, response surface methodology, Box-Behnken design

Posted Date: August 12th, 2020

DOI: https://doi.org/10.21203/rs.3.rs-51204/v1

License: (c) (i) This work is licensed under a Creative Commons Attribution 4.0 International License. Read Full License

Version of Record: A version of this preprint was published at Journal of Food Measurement and Characterization on February 1st, 2021. See the published version at https://doi.org/10.1007/s11694020-00805-6. 
1 Ultrasound-Assisted Extraction for Simultaneous Quantitation of Potential Sweetening

2 Compounds from Derris reticulata Aqueous Extracts: A Response Surface Methodology

3 Approach

4 Keerati Thamapan $^{\mathrm{a}}$, Natta Laohakunjit ${ }^{\mathrm{a}}$, Orapin Kerdchoechuen ${ }^{\mathrm{a}}$, Punchira Vongsawasdi ${ }^{\mathrm{b}}$,

5 and Withawat Mingvanish ${ }^{\mathrm{c}}$

6

7 Author information

8 a Division of Biochemical Technology, School of Bioresources and Technology,

$9 \quad$ King Mongkut's University of Technology Thonburi,

49 Tientalay 25 Rd., Takham, Bangkhuntien, Bangkok 10150, Thailand

11

${ }^{\mathrm{b}}$ Department of Microbiology, Faculty of Science, King Mongkut's University of Technology

Thonburi, 126 Pracha Uthit Rd., Bang Mod, Thung Khru, Bangkok 10140, Thailand

'Department of Chemistry, Faculty of Science, King Mongkut's University of Technology

Thonburi, 126 Pracha Uthit Rd., Bang Mod, Thung Khru, Bangkok 10140, Thailand

* Corresponding author. Tel.: +66 2470 7752; fax: +66 24707781.

E-mail address: nutta.lao@kmutt.ac.th (N. Laohakunjit). 


\section{Abstracts}

Background: Derris reticulata or Oi Sam Saun is a highly sweet Thai plant, rich in bioactive compounds, and widely used for its medicinal properties. In this study, sweet aqueous extracts from the stems of Oi Sam Saun were extracted using ultrasound-assisted extraction (UAE). Phenolic, flavonoid, and sugar compound extraction was optimized using the Box-Behnken design (BBD) and response surface methodology (RSM).

Methods: Three independent variables - extraction temperature $\left(40-80^{\circ} \mathrm{C}\right)$, sonication time (20-60 min), and extraction ratio $(1: 10-1: 30 \mathrm{~g} / \mathrm{mL})$-were investigated, and the optimal condition was used to determined phenolics, flavonoid and $18 \beta$-glycyrrhetinic acid by High performance liquid chromatography-diode array detector (HPLC-DAD). Sensory evaluation was also performed.

Results: The values of $84^{\circ} \mathrm{C}, 64 \mathrm{~min}$, and ratio $1: 8 \mathrm{~g} / \mathrm{mL}$ were found to be optimal. Under these conditions, experimental values were well correlated with predicted values, and phenolic, flavonoid, and sugar contents were determined as $0.4725 \mathrm{mgGAE} / \mathrm{gDW}, 0.1489 \pm 0.033$ $\mathrm{mgCE} / \mathrm{gDW}$, and $4.802 \pm 0.651 \mathrm{mg} / \mathrm{gDW}$, respectively. Gallic acid, p-coumaric acid, quercetin, and kaempferol was also found in optimal condition. Moreover, the extract contained $18 \beta$-glycyrrhetinic acid $(0.529 \pm 0.002 \mathrm{mg} / 100 \mathrm{mg})$ and was 166 times sweeter than sucrose.

Conclusion: High level of phenolics, flavonoids and sugars was detected in optimal condition of the extract. Therefore, this Thai medicinal plant, which has several pharmacological benefits, is newly potentially and applicable as a sweetening agent or sugar substitute in foods.

Keywords: ultrasound-assisted extraction, phenolics, flavonoids, 18ß-glycyrrhetinic acid, sweet Thai plant, response surface methodology, Box-Behnken design 


\section{Introduction}

Sweetness is one of five basic tastes and plays an important role in human diet. Most sweet tastes originate from sugars. However, excessive sugar intake can cause diseases, such as dental caries, hypertension, obesity, and diabetes (Malik et al. 2006). Currently, non-nutritive sweeteners and non-sugar sweetening agents are being consumed to avoid health problems associated with high sugar intake. Most non-nutritive sweeteners that are currently available globally are artificial sweeteners, such as aspartame, sucralose, saccharin, and acesulfame-K, some which have been reported to be harmful to life (Whitehouse et al. 2008). Many research attempts have been made to discover and develop natural non-nutritive sweetening compounds from plants. These attempts have been largely successful, but only a few of these compounds have been commercialized as sweeteners, including stevioside, rebaudioside, glycyrrhizin, mogroside, brazzein, and thaumatin. However, sweetening agents of plant origin, including phenolics, flavonoids, terpenoids, and sugars have both aglycone and glycone structures (Kim and Kinghorn et al. 2002). Some flavonoid or phenolic compounds are sweet, such as glycyphyllin, naringin dihydrochalcone, and dihydroquercetin 3-O-acetate (Kim and Kinghorn et al. 2002). A well-known low-calorie sweetener is glycyrrhizin, a glycosylated pentacyclic triterpenoid, containing one molecule of $18 \beta$-glycyrrhetinic acid, and two molecules of glucuronic acid. Additionally, the compound, 18ß-glycyrrhetinic acid, is found in Glycyrrhiza glabra L. or licorice (local name in Thai "Cha-em"). It is a widely used herbal medicine, native to southern Europe and parts of Asia, including Thailand (Khattak and Simpson, 2010). Thailand has a biodiversity of interesting plants such as "Oi Sam Saun" or "Cha em Nua" (Derris reticulata Craib.), found in semi-shaded areas of dry evergreen forests, the edge of evergreen mixed (dipterocarp) forests, bamboo forests, or along streams (50-450 m). The stems of this plant give a sweet taste similar to licorice; it is used as a sweetener in local medicine and as a laxative (Sirichamorn et al. 2012). Although some flavonoids, such as lupinifolin, 
dereticulatin, and pyranoflavonone, were reported in Oi Sam Saun (Yusook et al. 2017; Mahidol et al. 1997; Mahidol et al. 2002), these compounds did not show sweet tastes.

Generally, sweetening compounds were extracted via conventional methods, such as maceration, soxhlet extraction, and supercritical fluid extraction. Xia et al. (2008) extracted sweetening components from Siraitia grosvenorii or monk fruit by soxhlet extraction method. Further, Koh et al. (2009) extracted sweetening compounds from Chinese sweet tea plant (Rubus suavissimus S. Lee) by soaking in water and precipitating with alcohol. Choi et al. (2002) extracted stevioside from stevia leaves by supercritical fluid extraction. Extraction is a crucial step for the isolation of bioactive sweetening compounds from plant materials. However, conventional methods have certain limitations, such as low yield, too much solvents, and bitter tastes caused by the materials used (Armenta et al. 2008). Therefore, modern techniques, such as ultrasound-assisted extraction (UAE), microwave assisted extraction, and pressurized liquid extraction, are modified approaches that have significant advantages over conventional methods (2006). These techniques have been applied in extracting commercial natural sweeteners, such as stevioside, glycyrrhizin, and mogroside (Pan et al. 2008; Rao et al. 2012; Charpe and Rathod, 2012).

UAE has received considerable attention as a promising alternative to conventional methods (Maran and Priya, 2016). It has been applied in several research and development fields, including phytochemical product extraction and the food industry (Esclapez et al. 2011). It is a simple, low cost, and highly effective technique that exhibits a high efficiency yield in a short time (Al-Dhabi et al. 2017). According to some reports, UAE has been applied for the extraction of natural sweeteners; Charpe and Rathod (2012) extracted glycyrrhizic acid from licorice root using ultrasound, and Liu et al. (2010) extracted total carbohydrate and rebuadioside A from stevia leaves by UAE. 
However, the UAE method needs to be optimized depending on several factors that can influence the phytochemical extraction yield, including the extraction temperature, ultrasonic time, solvent composition, particle diameter, liquid-solid ratio, and electrical acoustic intensity (Sheng et al. 2017). Currently, response surface methodology (RSM) is a mathematical tool widely used in the research and food industry. Its advantages include decreasing the number of experimental runs, evaluating the effect of several variables, and optimizing conditions. Among many classes of RSM designs, Box-Behnken designs (BBDs) are a class of rotatable or nearly rotatable second-order designs that are based on three-level incomplete factorial designs. BBDs are slightly more efficient with fewer experimental runs than the central composite designs (CCDs) (Ferreira et al. 2007).

To the best of our knowledge, there is a lack of information on sweetening compounds and their related compounds from Derris reticulata Craib., and there are only few reports on suitable extraction methods for these compounds. Therefore, this study was aimed to extract such compounds (phenolics, flavonoids, and sugars) from Oi Sam Saun using UAE, optimizing them using RSM, and investigating their sweetness potent.

\section{Materials and Methods}

\section{Chemicals}

Folin-Ciocalteu reagent, sodium carbonate, methanol, acetonitrile, and formic acid were purchased from Sigma (Singapore). All solvents and standard compounds were of HPLC grade and all chemicals were of analytical grade.

\section{Plant materials}

Derris reticulata Craib. (Leguminosae) or Oi Sam Saun stems were collected in July, 2015 from central regions of Thailand. Botanical identification was graciously performed by Assoc. 
123

124

Prof. Saranya Vajrodaya of the Faculty of Botany, Kasetsart University, Bangkok, Thailand. Voucher specimen (BK no. 069447) was then deposited at the Forest Herbarium-BKF, Bangkok, Thailand. Stems were dried at $50^{\circ} \mathrm{C}$ until they attained constant weight $(12 \%$ moisture content), ground to powder, sieved through a 40-mesh sieve, and kept at room temperature prior to the experiments.

\section{Extraction of Oi Sam Saun by Ultrasound-assisted extraction (UAE)}

UAE was carried out using an ultrasonic bath (Elmasonic E70H) set to $120 \mathrm{~W}$ and $37 \mathrm{~Hz}$. Plant powder was extracted using distilled water at different extraction temperatures $(40,60$, and $\left.80^{\circ} \mathrm{C}\right)$, times $(20,40$, and $60 \mathrm{~min})$, and ratios $(1: 10,1: 20$ and 1:30 w/v). At designated extraction intervals, the mixture was taken and filtered with Whatman No. 1 filter paper; the Oi Sam Saun UAE extracts were collected to determine the yield, colour, and sweetening compounds.

Determination of extraction yield, colour, and sweetening compounds in Oi Sam Saun extract

\section{Extraction yield}

Oi Sam Saun UAE extracts were collected, the water was removed under vacuum using a rotary evaporator (Buchi, Germany) at $40^{\circ} \mathrm{C}$, and they were freeze-dried. The percentage yield of the dried crude extracts was determined and calculated according to Equation 1:

$\%$ yield $=($ weight of crude extract/weight of dried sample $) \times 100$

Equation 1

\section{Colour measurement}

Colours of the Oi Sam Saun UAE extracts were measured using a HunterLab colorimeter (Miniscan EZ; Hunter Associates Laboratory Inc., Reston, USA). Each sample was measured in triplicates and analysed using the CIE $L^{*} a^{*} b^{*}$ system. Here, $L^{*}$ represents the lightness of the 
colours from 0 (dark) to 100 (light), $a^{*}$ represents the greenness/redness parameter (negative $a^{*}$ is green and positive $a^{*}$ is red), and $\mathrm{b} *$ represents the grade of blueness/yellowness (negative $b^{*}$ is blue and positive $b^{*}$ is yellow). The angular coordinates of the hue angle $\left(h^{\circ}\right)$ were calculated according to Equation 2:

$$
\begin{array}{lr}
h^{\circ}=\tan -1\left(b^{*} / a^{*}\right) \text { when } a^{*}>0 \text { and } b^{*}>0 & \text { Equation 2a } \\
h^{\circ}=180^{\circ}+\tan -1\left(b^{*} / a^{*}\right) \text { when } a^{*}<0 & \text { Equation 2b } \\
h^{\circ}=360^{\circ}+\tan -1\left(b^{*} / a^{*}\right) \text { when } a^{*}>b^{*} \times \text { and } b^{*}<0 & \text { Equation 2c }
\end{array}
$$

For the browning index (BI) of Oi Sam Saun UAE extracts, BI was calculated using Equations $3 \mathrm{a}$ and $3 \mathrm{~b}$ :

$$
B I=\frac{100(x-0.31)}{0.172} \quad \text { Equation } 3 \mathrm{a}
$$

Where:

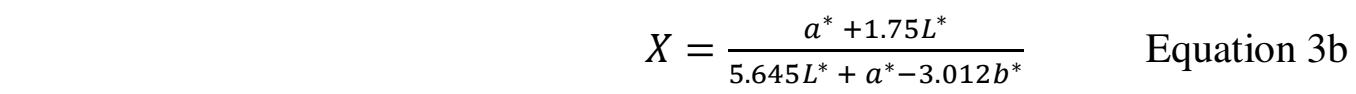

The browning index (BI) represents the purity of the brown colour or brown pigment concentration (Guerrero et al. 1996; Palou et al. 1999).

\section{Total phenolic content}

Phenolic content was determined using the Folin-Ciocalteu method described by Gonçalves et al. (2013). Folin reagent $(2.5 \mathrm{~mL}$, diluted $10 \times)$ was added to $0.5 \mathrm{~mL}$ of the extract, to which $2 \mathrm{~mL}$ sodium carbonate $(5 \mathrm{~g} / \mathrm{L})$ was then added. The mixture was then placed in the dark for $1 \mathrm{hr}$ before the absorbance was read at $760 \mathrm{~nm}$. Gallic acid was used as a reference standard and the total phenolic content was expressed as gallic acid equivalents (GAE, mg/g extract).

\section{Total flavonoid content}


172 Total flavonoids were determined using a spectrophotometric assay developed by Dini (2011).

173 About $0.5 \mathrm{~mL}$ of the extract and catechin standard $(20-100 \mathrm{mg} / \mathrm{L})$ were mixed in a test tube with $2 \mathrm{~mL}$ of distilled water, and $5 \%$ sodium nitrite $(0.15 \mathrm{~mL})$ was added to the test tube. After

$1755 \mathrm{~min}, 10 \%$ aluminium chloride $(0.15 \mathrm{~mL})$ was added to the mixture. At $6 \mathrm{~min}, 1 \mathrm{M}$ sodium 176 hydroxide $(1 \mathrm{~mL})$ and distilled water $(1.2 \mathrm{~mL})$ were added, and the mixture was thoroughly mixed. The absorbance of the mixture was measured against a blank at $510 \mathrm{~nm}$. The total flavonoids of the extracts were expressed as mg catechin equivalent (CE)/g dry weight of the plant.

\section{Total sugar content}

Total sugar content was determined based on a colorimetric method (Dubois et al. 1956). The extract $(1 \mathrm{~mL})$ was mixed with $5 \%$ phenol $(1 \mathrm{~mL})$, and $5 \mathrm{~mL}$ of concentrated sulfuric acid was then added. The reaction mixture was incubated at room temperature for 20 minutes, and the absorbance was measured at $490 \mathrm{~nm}$ with glucose as standard. The total sugar content was expressed as mg glucose/g dry weight of plant (mg/g DW).

\section{Experimental design}

Box-Behnken experimental design (BBD) with three levels and three factors was selected to investigate the influence of process factors on RSM. The effects of three extraction factors (ratio, temperature, and sonication time) on three responses (phenolic, flavonoid, and sugar contents) were determined. Table 1 lists the original and code values of the extraction factors and their levels in the extraction process. The experimental design involves 17 experimental runs, including five replicates at centre points, used to allow for the estimation of a pure error sum of squares, and the total number of experimental runs were evaluated from the following equation (Maran and Priya, 2016). 
Where $\mathrm{K}$ is the number of experimental factors and $\mathrm{C}_{0}$ is the number of central points. model equation is:

$$
Y=\beta_{0}+\sum_{i=1}^{k} \beta_{i} X_{i}+\sum_{i=1}^{k} \beta_{i i} X_{i}^{2}+\sum_{i}^{k} \sum_{i}^{k} \beta_{j i} X_{i} X_{j}+\varepsilon
$$

Equation 5

202

Where $\mathrm{Y}$ is the dependent variable (flavonoid and phenolic), $\beta_{0}$ is the model constant, $\beta_{\mathrm{i}}, \beta_{\mathrm{ii}}$, $\beta_{\mathrm{ij}}$ are the model coefficients, $X$ is the independent variable, $k$ is the number of independent factors, and $\varepsilon$ is the error. The parameters, $\beta_{\mathrm{i}}, \beta_{\mathrm{ii}}, \beta_{\mathrm{ij}}$ represent the linear, quadratic, and interaction effects of the variables, respectively.

\section{Optimization of sweetening compound extraction conditions}

BBD experimental data were used to determine the optimal conditions for the model. All response variables (Total phenolic, flavonoid, and sugar contents) were kept at maximum, and the independent variables $\left(\mathrm{X}_{1}, \mathrm{X}_{2}\right.$, and $\left.\mathrm{X}_{3}\right)$ were kept within the desired range (between lower and higher level). Statistica 9.0 software (StatSoft Inc., Tulsa, OK) generated the optimal conditions based on BBD data. Further, the optimal condition gave the model a predicted value for each response, for comparison with the experimental value. Hence, the experiments were performed at the suggested optimal condition, and the response values obtained were compared with the model's predicted values.

\section{Determination of phenolic and flavonoid compounds}

Under optimal conditions, Oi Sam Saun UAE extract ( 10 mg) was hydrolysed using $10 \mathrm{~mL}$ of $1 \mathrm{M}$ trifluoroacetic acid (TFA) at $90^{\circ} \mathrm{C}$ for $60 \mathrm{~min}$. Next, the solution was mixed with 40 $\mathrm{mL}$ methanol and taken to the rotary evaporator at $40^{\circ} \mathrm{C}$, until the remaining TFA was removed. 
The hydrolysed sample was mixed with $20 \mathrm{~mL}$ deionised water and filtered with a $0.45 \mu \mathrm{m}$ syringe filter used for phenolic and flavonoid determination.

Phenolic and flavonoid compounds were determined under optimal Oi Sam Saun UAE conditions. The extracts were analysed using an HPLC-DAD (diode array detector; 1200 Series, Agilent Technologies, USA), using an Eclipse XDB-C18 column (4. 6 mm ID x 250 $\mathrm{mm}, 5 \mu \mathrm{m}$ ) and a linear gradient with water (pH 2.5) containing TFA (A) and acetonitrile (B) for $65 \mathrm{~min}$ at a flow rate of $1.0 \mathrm{~mL} / \mathrm{min}$. The gradient was set as follows: $0-20 \mathrm{~min}, 95-90 \%$ A; 20-50 min, $95-70 \%$ A; 50-55 min, 70-50\% A; 55-60 min, 50-95\% A; 60-65, 95\% A. The samples $(5 \mu \mathrm{L})$ were loaded into the HPLC-DAD. The DAD detector was set at 280 and $350 \mathrm{~nm}$ for phenolic and flavonoid detection, respectively.

\section{Sugar analysis}

Sugar type and content were identified by high performance liquid chromatographyevaporating light scattering detector (HPLC-ELSD; Alltech, Buchi, Switzerland). Oi Sam Saun UAE extracts, under optimal conditions, were dissolved in distilled water at a concentration of $1,000 \mathrm{ppm}$ and filtered through $0.45 \mu \mathrm{m}$ (Millipore) filters. Separation was achieved using a Rezex RPM Monosaccharide column ( $300 \mathrm{~mm} \times 7.8 \mathrm{~mm}$ ID, $8 \mu \mathrm{m}$ particle size). The mobile phase was water in isocratic elution with a flow rate of $0.6 \mathrm{~mL} / \mathrm{min}$ for $30 \mathrm{~min}$. The detection of analytes was carried out using an evaporative light scattering detection (Alltech, Buchi, Switzerland) technique which detects organic molecules by mass; hence, it is useful in the quantitative determination of non-UV-sensitive compounds. The drift tubes for ELSD were set at $105^{\circ} \mathrm{C}$ and the flow rate of nebulizing gas $\left(\mathrm{N}_{2}\right)$ was 2.6 standard litre per minute (SLM). The sugar standard chromatograms were for glucose, fructose, and sucrose.

\section{Determination of 18ß-glycyrrhetinic acid}


246 The 18ß-glycyrrhetinic acid content of the Oi Sam Saun UAE extract, under optimal

247 conditions, was identified by the modified method of Esmaeili et al. (2010), using 1200 series

248 Agilent HPLC system with a $20 \mu \mathrm{L}$ sample loop attached to a DAD. HPLC analysis was 249 completed using a reversed phase XDB-C18 column $(250 \times 4.6 \mathrm{~mm}, 5 \mathrm{~mm})$ and $18 \beta$ 250 glycyrrhetinic acid was determined using an acetonitrile/phosphoric acid $(3 / 1, \mathrm{v} / \mathrm{v} ; \mathrm{pH}=2.5)$ mobile phase at flow rates of $1.0 \mathrm{~mL} / \mathrm{min}(0-8 \mathrm{~min})$ and $0.6 \mathrm{~mL} / \mathrm{min}(8-20 \mathrm{~min})$ at a detector wavelength of $230 \mathrm{~nm}$.

\section{Sweetness potency by sensory evaluation}

\section{Sensory evaluation of Oi Sam Saun UAE aqueous extracts}

Sensory evaluation of Oi Sam Saun UAE extracts, under optimal conditions, was conducted by 10 semi-trained panellists ( 3 males and 7 females) aged $24-30$ years old. Sensory profiling was performed by selecting extracts from the best condition. The intensity of each attribute, including sweet, sour, and bitter tastes, was scored on a scale of 1 to 9 . Sucrose, citric acid, and caffeine were used as standards for sweet, sour, and bitter tastes, respectively. The individual panellists who took part in the study were trained before the tasting and ranking test. The sweet solution $(10 \mathrm{~mL})$, at a concentration of $1,2,5$, and $10 \%$ sucrose, was selected for the first session, and $0.5,1,2$, and $3 \%$ sucrose were used for training in the second session. UAE aqueous extracts $(1 \mathrm{~mL})$ were served in opaque disposable plastic cups at room temperature (Meilgaard et al. 2006).

Sweetness intensity estimation

The sweetness potency of each sample, relative to that of sucrose, was calculated using the following formula: 
271 Where $\mathrm{A}$ is the concentration $(\% \mathrm{w} / \mathrm{v})$ of sample solution at $10 \%$, and $\mathrm{B}$ is the concentration

$272(\% \mathrm{w} / \mathrm{v})$ of sucrose with the same sweetness as sample. B was calculated from the linear regression formula of the sweetness score (1-9 point) against sucrose concentration ( $0-15 \%$ w/v) (Yoshikawa et al. 2002; Darise et al. 1984).

\section{Statistical analysis}

277 All tests were performed in triplicate. Statistical analysis was performed by the analysis of variance (ANOVA) and least significant difference (LSD) test, using SAS statistical software, version 9 (SAS Institute Inc., Cary, NC, USA), at 95\% confidential interval or probability at $\leq$ 0. 05. The three-dimensional (3D) response surface plots of the experimental model were generated using the Statistica 9.0 program (StatSoft Inc., Tulsa, OK).

\section{Results and discussion}

\section{Fitting the response surface model}

The preparation process to prepare Oi Sam Saun UAE extracts and overall experiment is illustrated in Fig. 1. In this study, UAE involved three important parameters-temperature, sonication time, and extraction ratio- - which can strongly influence the amount of yield, colour, and phenolic, flavonoid, and sugar contents of Oi Sam Saun extracts. Experiments were applied to determine the optimum temperature, time, and extraction ratio for yield, colour, and phenolic, flavonoid, and sugar compounds in the extract, based on BBD. However, the extraction yield, colour ( $\mathrm{L}^{*} \mathrm{a}^{*}, b^{*}$, and $h^{\circ}$ ), and browning index (BI) for all crude Oi Sam Saun extracts showed no significant difference ( $p>0.05$; data not shown). Additionally, there was no correlation between yield, colour, browning index, and phenolic, flavonoid, and sugar contents because statistical analysis of the results revealed no significant difference. Moreover, some phenolic and 
296

297

298

299

300

301

302

303

304

305

306

307

308

309

310

311

312

313

314

315

316

317

318

319

320

browning index of Oi Sam Saun UAE extracts were not different under every extraction condition. Therefore, phenolic, flavonoid and sugar contents were selected to determine the optimal condition for the Oi Sam Saun UAE process.

The experimental results for the 17 experimental points included five central points from the BBD, which were calculated from Equation 5, and are shown in Table 1. The quadratic model was applied to show the influence of variables over phenolic, flavonoid, and sugar contents in the extract. The quadratic model regression analysis equations, which were calculated from Equation 6 of Oi Sam Saun, were obtained. The results showed that the experimental data fit a quadratic model in the phenolic, flavonoid, and sugar contents of Oi Sam Saun based on ANOVA (Table 2), with significant $R^{2}$ values (>0.90) for the effect of extraction temperature, sonication time, and extraction ratio on phenolic, flavonoid, and sugar contents. The secondorder equations for these variables are shown as Equation 7 to 9, respectively.

Phenolic $(\mathrm{mg} / \mathrm{g})=0.233+0.028 \mathrm{X}_{1}+0.027 \mathrm{X}_{2}-0.147 \mathrm{X}_{3}+0.039 \mathrm{X}_{1} \mathrm{X}_{2}+0.056 \mathrm{X}_{2}^{2}+$ $0.089 X_{3}^{2}$

Equation 7

11

Flavonoid $(\mathrm{mg} / \mathrm{g})=0.037+0.003 \mathrm{X}_{1}+0.002 \mathrm{X}_{2}-0.058 \mathrm{X}_{3}+0.004 \mathrm{X}_{1}^{2}+0.002 \mathrm{X}_{1} \mathrm{X}_{2}-$

$0.002 \mathrm{X}_{1} \mathrm{X}_{3}-0.001 \mathrm{X}_{2} \mathrm{X}_{3}+0.003 \mathrm{X}_{2}^{2}+0.027 \mathrm{X}_{3}^{2}$

Equation 8

$$
\operatorname{Sugar}(\mathrm{mg} / \mathrm{g})=2.010+0.140 \mathrm{X}_{1}-1.415 \mathrm{X}_{3}-0.210 \mathrm{X}_{2} \mathrm{X}_{3}+0.741 \mathrm{X}_{3}^{2} \quad \text { Equation } 9
$$

where $\mathrm{X}_{1}$ : temperature $\left({ }^{\circ} \mathrm{C}\right), \mathrm{X}_{2}$ : sonication time $(\mathrm{min})$, and $\mathrm{X}_{3}$ : solid/liquid ratio $(\mathrm{mL} / \mathrm{g})$. ANOVA was used to evaluate the significance of the quadratic polynomial models. The linear terms of temperature $\left(\mathrm{X}_{1}\right)$, time $\left(\mathrm{X}_{2}\right)$, and ratio $\left(\mathrm{X}_{3}\right)$ showed a significant effect $(p \leq 0.05)$ on phenolic content. The quadratic terms of time $\left(\mathrm{X}_{2}^{2}\right)$ and ratio $\left(\mathrm{X}_{3}^{2}\right)$ on phenolic content also 
exhibited a significant effect $(p \leq 0.05)$, whereas the effect of temperature was insignificant $(p$ $>0.05)$. The combined effect on phenolic contents was significantly $(p \leq 0.05)$ influenced by temperature and time.

The linear and quadratic effects of all three parameters on flavonoid content were significant $(p \leq 0.05)$. ANOVA showed that, in combination, flavonoid contents were significantly influenced by temperature $\left(\mathrm{X}_{1}\right)$ and time $\left(\mathrm{X}_{2}\right)(p \leq 0$.05). Moreover, in combination, flavonoid content was also significantly influenced by extraction temperature $\left(\mathrm{X}_{1}\right)$ and ratio $\left(\mathrm{X}_{2}\right)$, and time $\left(\mathrm{X}_{1}\right)$ and ratio $\left(\mathrm{X}_{3}\right)(p \leq 0.05)$.

The linear effects of temperature $\left(\mathrm{X}_{1}\right)$ and ratio $\left(\mathrm{X}_{3}\right)$, and the quadratic effect of ratio $\left(\mathrm{X}_{3}^{2}\right)$ on sugar content in the Oi Sam Saun UAE extract was significantly different $(p \leq 0.05)$. In combination, only the interaction between time and ratio had a significant $(p \leq 0.05)$ effect on sugar content.

Moreover, the highest values of estimated regression coefficients for extraction ratio $\left(\beta_{3}=-0.147,-0.058\right.$, and -1.415$)$ indicated that it was the most important linear variable influencing phenolic, flavonoid, and sugar contents. The negative value implied that phenolic, flavonoid, and sugar contents increased with decreasing extraction ratio. In addition, the model fitness was investigated using the lack-of-fit test $(p \leq 0.05)$, which indicated the suitability of models for accurate prediction of the variation (Yolmeh et al. 2014).

\section{Optimization of the extraction process}

The 3D response surface plots and two-dimensional (2D) contour plots were constructed from the regression equations to visualize and study the relationship between the response (temperature, sonication time, and extraction ratio) and sweetening compound (phenolic, flavonoid, and sugar) extraction variables shown in Fig. 2. 
For phenolic compounds in the Oi Sam Saun extracts, the 3D and 2D RSM plots are shown as a function of temperature and sonication time in Fig. 2a. ANOVA results indicated that $\mathrm{X}_{1}$ (temperature) and $\mathrm{X}_{2}$ (sonication time) had positive interaction effects on phenolic content ( $p \leq 0.05$; Table 2). Phenolic content increased with increasing extraction temperature and time, probably due to enhanced mass transfer rate and diffusibility at higher temperatures of solvent into the plant cell matrix. Further, the solubility of phenolic compounds also increased at higher temperatures. These phenomena create bubble cavitation in liquid medium and cause bubble collapse, which damages the plant cell matrix (Charpe and Rathod, 2012; Esclapez et al. 2011). It can be concluded that the maximum phenolic content of Oi Sam Saun UAE extract was attained when extraction temperature and sonication time were $\sim 84^{\circ} \mathrm{C}$ and 64 min, respectively.

The 3D and 2D RSM plots of Oi Sam Saun flavonoid contents are shown in Fig. 2b-

2d. Based on ANOVA, the combined effect of temperature and sonication time, temperature and ratio, and sonication time and ratio was statistically significant $(p \leq 0.05)$. The flavonoid content increased with increasing temperature and sonication time (Fig. 2b). Increasing the sonication time influenced flavonoid content due to swelling, and hydration of plant material could be accelerated by the cavitation effect of ultrasound waves during the initial extraction period. The asymmetric collapse of micro-bubbles near surfaces was also associated with micro-jets that could cause the disruption and penetration of water into the matrix through temperature and extraction ratio and sonication time and extraction on flavonoid content, respectively. As seen in Fig. 2c, flavonoid content increased with increasing temperature and decreasing extraction ratio. Fig. 2d shows that increasing sonication time and decreasing extraction ratio increased flavonoid content. This phenomenon was also reported in the UAE 
of polyphenols from Sparganium stoloniferum (Wang et al. 2013). Taken together, it can be concluded that flavonoid content was the highest when the extraction ratio, temperature, and sonication time were $1: 8 \mathrm{~g} / \mathrm{mL}, 84^{\circ} \mathrm{C}$, and $64 \mathrm{~min}$, respectively.

For sugar compounds, Fig. 2e demonstrates the interactive effect of sonication time and extraction ratio on sugar content. ANOVA showed that sugar content was dependent on sonication time and extraction ratio, combined $(p \leq 0.05)$. Sugar content increased with increasing sonication time and decreasing extraction ratio. According to Fig. 2e, the longer the sonication time, the higher is the sugar content, which is similar to the phenolic and flavonoid contents. Therefore, sugar content was the highest when the extraction ratio and sonication time were around $1: 8 \mathrm{~g} / \mathrm{mL}$ and $64 \mathrm{~min}$, respectively.

\section{Prediction of optimal conditions}

The numerical optimization method was used to optimize the UAE conditions. The optimal Oi Sam Saun UAE conditions, which gave the maximum phenolic, flavonoid, and sugar content were 1.2 (temperature), 1.2 (sonication time), and -1.2 (extraction ratio), in coded form. The corresponding actual optimum extraction conditions were $84^{\circ} \mathrm{C}, 64 \mathrm{~min}$, and $1: 8 \mathrm{~g} / \mathrm{mL}$, respectively. Under these conditions, the predicted values for phenolic, flavonoid, and sugar contents were $0.4725 \mathrm{mg} / \mathrm{g}, 0.1688 \mathrm{mg} / \mathrm{g}$, and $4.802 \pm 0.651 \mathrm{mg} / \mathrm{g}$, respectively (Table 3).

\section{Phenolic and flavonoid profile of optimized Oi Sam Saun UAE extract}

Some phenolics and flavonoids have been reported for their sweetening properties (Kim and Kinghorn et al. 2002). Thus, the determination and identification of phenolic and flavonoid compounds in Oi Sam Saun UAE extract was done. Under optimized UAE conditions, the extract of Oi Sam Saun $\left(84^{\circ} \mathrm{C}\right.$, and $\left.64 \mathrm{~min}, 1: 8 \mathrm{~g} / \mathrm{mL}\right)$ was investigated in the presence of phenolic and flavonoid compounds. The compounds were identified by comparing their 
retention times and UV absorption spectrum with those of standards. Eight compounds were shown in the chromatogram, but only four compounds were identified and quantified - two phenolics (gallic acid, $0.108 \pm 0.012 \mathrm{mg} / \mathrm{g}$ extract and p-coumaric acid, $0.082 \pm 0.007 \mathrm{mg} / \mathrm{g}$ extract) and two flavonoids (quercetin, $0.047 \pm 0.008 \mathrm{mg} / \mathrm{g}$ extract and kaempferol, $0.031 \pm$ $0.002 \mathrm{mg} / \mathrm{g}$ extract) (Fig. 3; Table 4). These four compounds, found in the Oi Sam Saun UAE extract, are not typically present in free form and mostly give a bitter, rather than sweet taste. However, quercetin and kaempferol structures might have similar dihydroflavonol or glycoside forms as other sweet phenolic and flavonoid compounds, such as glycyphyllin, a dihydrochalcone glycoside, or $(2 R, 3 R)$-dihydroquercetin 3-O-acetate, a dihydroflavonol (Kim and Kinghorn et al. 2002), thereby giving a sweet taste to Oi Sam Saun extract. Hence, the unknown phenolic and flavonoid compounds may be the key to the sweet taste of this plant extract.

\section{Sugar profile of Oi Sam Saun UAE extract}

The sugar composition of the optimized Oi Sam Saun UAE extract was quantified via HPLCELSD. Glucose, fructose, sucrose, and maltose were used as sugar standards. The chromatogram results showed that the extract (Fig. 4) contained known sugars including sucrose $(1.333 \pm 0.098 \mathrm{mg} / \mathrm{g}$ extract $)$, glucose $(0.705 \pm 0.051 \mathrm{mg} / \mathrm{g}$ extract $)$, and fructose $(0.891$ $\pm 0.074 \mathrm{mg} / \mathrm{g}$ extract), and three unknown sugars (Table 4). The type and concentration of sugars were related to the sweetness intensity of the plant. Glucose in this plant might be in the form of glycoside and impart a sweet taste, like glycyphyllin, which has glucose in its structure. Sucrose also gives a sweet taste to this plant, depending on the concentration. Therefore, analysis of the phenolic, flavonoid, and sugar components suggested that phenolic and flavonoid compounds in Oi Sam Saun UAE extract exist in glycoside forms, which might be responsible for imparting a sweet taste to it (Kim and Kinghorn et al. 2002). 


\section{The 18ß-glycyrrhetinic acid content of optimized Oi Sam Saun UAE extract}

422

The chromatogram in Fig. 4 shows that the $18 \beta$-glycyrrhetinic acid content was $0.529 \pm 0.002$ mg/100 mg of optimized Oi San Saun UAE extract (0.529\%; Table 4). The typical amount of $18 \beta$-glycyrrhetinic acid found in licorice root was $0.1-1.6 \%$, depending on the region of cultivation and species (Sabbioni et al. 2006). Moreover, 18ß-glycyrrhetinic acid gives a sweet taste, but binds to two glucuronic acid molecules. Thus, $18 \beta$-glycyrrhetinic acid, found in Oi Sam Saun UAE extract, might be bound to some sugars (such as glucose or fructose), forming some of the unidentifiable compounds from the sugar profile, and possesses a sweet taste. This result is consistent with the sensory profile, which showed that the optimized Oi San Saun UAE extract gave a sweet taste. 18ß-glycyrrhetinic acid has been shown to possess several pharmacological benefits, such as an antiulcerative effect, anti-inflammatory activity, direct and indirect antiviral activity, interferon inducibility, an antihepatitis effect, and an antihyperglycemic effect (Kalaiarasi et al. 2009).

\section{Sweetness intensity of optimized Oi Sam Saun UAE extracts}

The optimized Oi Sam Saun UAE extract $\left(84^{\circ} \mathrm{C}, 64 \mathrm{~min}, 1: 8 \mathrm{~g} / \mathrm{mL}\right)$ was selected for sensory test. The sensory scores for each stimulus (sweetness, sourness, and bitterness) on a given adjective scale were detected as 6.94, 1.26, and 0.24, respectively (Table 3).

The relationship between the sweetness score from the individual panellists who took part in the study and the sucrose concentration was determined, and the equation, $y=0.6981 \mathrm{x}$ $\left(R^{2}=0.9656\right)$, was generated, and then used to calculate the B value in Equation 6. The Oi Sam Saun extract was 166 times sweeter than sucrose, which could be attributed to the combined effect of several compounds in the plant's crude extracts, such as polyphenols, alkaloids, and other pigments, which contribute to the sweet taste and concentration of 
sweetening compounds in the crude extracts. Polyphenols are responsible for some important sensory properties associated with foods. This complex and large family of molecules is responsible for the production of taste sensations, ranging from bitter to astringent and pungent, depending on the polyphenol composition of the food (Kaushik et al. 2010). Therefore, the different concentrations of phytochemical compounds, including flavonoids, sugars, and phenolics, elicited similar or better sensory responses from the individual panellists who took part in the study. The presence of phenolic compounds adversely influenced the acceptability of the extracts. In contrast, the presence of flavonoids and sugars enhanced the overall preference for Oi Sam Saun UAE extracts. High concentrations of these compounds may also play a beneficial role when incorporated as part of sweeteners since they contribute to antioxidant activity inherent in UAE extracts.

\section{Conclusion}

For the first time, the phenolic, flavonoid, and sugar compounds from Oi Sam Saun were extracted using UAE. This BBD (three levels, three factors) with RSM optimization study showed that UAE gives the best yield of sweetening compounds. The extraction yield, colour, and browning index of Oi Sam Saun UAE extracts were similar under all extraction conditions. The optimal conditions for Oi Sam Saun extraction were: $84^{\circ} \mathrm{C}, 64 \mathrm{~min}$ and 1:8 g/mL, and the maximum phenolic, flavonoid, and sugar yields were, 0.4725 mgGAE/gDW, 0. $1489 \pm$ $0.033 \mathrm{mgCE} / \mathrm{gDW}$, and $4.802 \pm 0.651 \mathrm{mg} / \mathrm{gDW}$, respectively. The best extraction conditions yielded $0.108 \pm 0.012 \mathrm{mg} / \mathrm{g}$ gallic acid, $0.082 \pm 0.007 \mathrm{mg} / \mathrm{g} p$-coumaric acid, $0.047 \pm$ $0.008 \mathrm{mg} / \mathrm{g}$ quercetin, and $0.031 \pm 0.002 \mathrm{mg} / \mathrm{g}$ kaempferol. Sucrose, glucose, and fructose concentrations were $1.333 \pm 0.098,0.705 \pm 0.051$ and $0.891 \pm 0.074 \mathrm{mg} / \mathrm{g}$ extract, respectively. The amount of $18 \beta$-glycyrrhetinic acid was $0.529 \pm 0.002 \mathrm{mg} / 100 \mathrm{mg}$ of extract. Moreover, the Oi Sam Saun UAE extract was 166 times sweeter than sucrose. These results reveal the 
470

471

472

473

474

475

476

477

478

479

480

481

482

483

484

485

486

487

488

489

490

491

492

493

potential application of this Thai medicinal plant in foods as a sweetening agent to substitute sugars and provide several pharmacological benefits. Moreover, its application as a sweetening compound may be rationalized for suitable processing to improve taste and sweetness potency. However, the fractionation, purification, and identification processes of individual sweetening compounds need to be investigated. Therefore, further studies should focus on identifying the new sweetening compounds via LC-MS-MS.

\section{Abbreviations}

UAE: ultrasound-assisted extraction; BBDs: Box-Behnken designs; RSM: response surface methodology; HPLC-DAD: high performance liquid chromatography-diode array detector; GAE: gallic acid equivalents; CE: catechin equivalent; CCDs: central composite designs; $\mathrm{BI}$ : browning index; TFA: trifluoroacetic acid; HPLC-ELSD: high performance liquid chromatography-evaporating light scattering detector; SLM: standard litre per minute; ANOVA: analysis of variance; and LSD: least significant difference; 3D: three-dimensional; 2D: two-dimensional.

\section{Authors' Contributions}

KT designed the study and reviewed manuscript concept, structure, topics, performed the research experiments, interpreted the results, acquired the data and wrote the manuscript with original draft, review \& editing. NL as supervision designed, reviewed and developed manuscript concept, structure and topics. OK, PV and WM were involved in the analysis. All authors read and approved the final manuscript of publication.

\section{Acknowledgements}


494 The authors are thankful to Thailand Research Fund through the Royal Golden Jubilee Ph.D.

495 Program (Grant No. PHD/0073/2554), King Mongkut's University of Technology Thonburi

496 (KMUTT), Bangkok, Thailand for providing all the necessary facilities to carry out the research

497 work.

498

499 Competing interest

500 The authors declare that they have no competing interests.

501

502 Availability of data and materials

503 All data that are relevant to the study are reported within the article.

504

505 Consent for publication

506 The authors approved the consent for publishing the manuscript.

507

508 Ethics approval and consent to participate

509 All the authors have read and agreed the ethics for publishing the manuscript. The study was 510 conducted in compliance with the ethical principles stated in the ethical guideline in human

511 experiments from King Mongkut's University of Technology Thonburi (2018) and the protocol 512 for this study. The appropriateness of this study was reviewed and approved by the ethics 513 committee of each participating facility. Written informed consent was obtained from all 514 participants in this study.

515

$516 \quad$ Funding 
517 This work was financially supported by Thailand Research Fund through the Royal Golden

518 Jubilee Ph.D. Program (Grant No. PHD/0073/2554), King Mongkut's University of

519 Technology Thonburi (KMUTT), Bangkok, Thailand.

520

\section{References}

522 Al-Dhabi NA, Ponmurugan K, Jeganathan PM (2017) Development and validation of ultrasound-assisted solid-liquid extraction of phenolic compounds from waste spent coffee grounds. Ultrason Sonochem 34:206-213.

525

Armenta S, Garrigues S, De la Guardia M (2008) Green analytical chemistry. Trac-Trend Anal

528

Charpe TW, Rathod VK (2012) Extraction of glycyrrhizic acid from licorice root using ultrasound: process intensification studies. Chem Eng Process 54:37-41.

Choi YH, Kim I, Yoon KD, Lee SJ, Kim CY, Yoo KP, Choi YH, Kim J (2002) Supercritical 533 fluid extraction and liquid chromatographic-electrospray mass spectrometric analysis of stevioside from Stevia rebaudiana leaves. Chromatographia 55:617-620.

Darise M, Mizutani K, Kasai R, Tanaka O, Kitahata S, Okada S, Ogawa S, Murakami F, Chen FH (1984) Enzymic transglucosylation of rubusoside and the structure-sweetness relationship of steviol-bisglycosides. Agric Biol Chem 48:2483-2488.

539

540 Dini I (2011) Flavonoid glycosides from Pouteria obovata (R. Br.) fruit flour. Food Chem $541 \quad 124: 884-888$. 
543 Dubois M, Gilles KA, Hamilton JK, Rebers PT, Smith F (1956) Colorimetric method for 544 determination of sugars and related substances. Anal Chem 28:350-356.

545

546 Esclapez M, García-Pérez JV, Mulet A, Cárcel J (2011) Ultrasound-assisted extraction of 547 natural products. Food Eng Rev 3:108.

548

549 Esmaeili S, Naghibi F, Mosaddegh M, Nader N (2010) Determination of 18ß-glycyrrhetinic 550 acid in Glycyrrhiza glabra L. extract by HPLC. Iran J Pharm Sci 137-141.

551

552 Ferreira SC, Bruns R, Ferreira H, Matos G, David J, Brandao G, da Silva EP, Portugal L, Dos 553 Reis P, Souza A (2007) Box-Behnken design: an alternative for the optimization of analytical 554 methods. Anal Chim Acta 597:179-186.

555

556

Gonçalves S, Gomes D, Costa P, Romano A (2013) The phenolic content and antioxidant 557 activity of infusions from Mediterranean medicinal plants. Ind Crops Prod 43:465-471.

558

559

Guerrero S, Alzamora S, Gerschenson L, (1996) Optimization of a combined factors 560 technology for preserving banana purée to minimize colour changes using the response surface 561 methodology. J Food Eng 28:307-322.

562

563 Kalaiarasi P, Pugalendi KV (2009) Antihyperglycemic effect of $18 \beta$-glycyrrhetinic acid, 564 aglycone of glycyrrhizin, on streptozotocin-diabetic rats. Eur J Pharmacol 606:269-273. 
566 Kaushik R, Narayanan P, Vasudevan V, Muthukumaran G, Usha A 2010. Nutrient composition

567 of cultivated stevia leaves and the influence of polyphenols and plant pigments on sensory and 568 antioxidant properties of leaf extracts. Int J Food Sci Tech 47:27-33.

569

570 Kelebek H, Canbas A, Jourdes M, Teissedre PL (2010) Characterization of colored and 571 colorless phenolic compounds in Öküzgözü wines from Denizli and Elazig regions using HPLC-DAD-MS. Ind Crops Prod 31:499-508.

573

Khattak KF, Simpson TJ (2010) Effect of gamma irradiation on the antimicrobial and free radical scavenging activities of Glycyrrhiza glabra root. Radiat Phys Chem 79:507-512.

576

577 Kim NC, Kinghorn AD (2002) Highly sweet compounds of plant origin. Arch Pharm Res 25:725-746.

579

Koh GY, Chou G, Liu Z (2009) Purification of a water extract of Chinese sweet tea plant 581

(Rubus suavissimus S. Lee) by alcohol precipitation. J Agric Food Chem 57:5000-5006.

582

583

Liu J, Li JW, Tang J (2010) Ultrasonically assisted extraction of total carbohydrates from Stevia rebaudiana Bertoni and identification of extracts. Food Bioprod Process 88:215-221.

Mahidol C, Prawat H, Kaweetripob W (2002) Two new pyranoflavanones from the stems

Derris reticulata. Heterocycles 57:1287-1292.

588

Mahidol C, Prawat H, Ruchirawat S, Lihkitwitayawuid K, Lin LZ, Cordell GA (1997)

590 Prenylated flavanones from Derris reticulata. Phytochemistry 45:825-829. 
591

592

Malik VS, Schulze MB, Hu FB (2006) Intake of sugar-sweetened beverages and weight gain:

593 a systematic review. Am J Clin Nutr 84:274-288.

594

595

Maran JP, Priya B (2016) Multivariate statistical analysis and optimization of ultrasound596 assisted extraction of natural pigments from waste red beet stalks. Int J Food Sci Tech 53:792597 799.

598

599

Meilgaard MC, Carr BT, Civille GV (2006) Sensory evaluation techniques. CRC press, 600 Florida.

601

602

Palou E, López-Malo A, Barbosa-Cánovas G, Welti-Chanes J, Swanson B (1999) 603 Polyphenoloxidase activity and color of blanched and high hydrostatic pressure treated banana 604 puree. J Food Sci 64:42-45.

605

606

Pan X, Liu H, Jia G, Shu YY (2000) Microwave-assisted extraction of glycyrrhizic acid from 607 licorice root. Biochem Eng J 5:173-177.

608

609

Rao AB, Reddy GR, Ernala P, Sridhar S, Ravikumar YV (2012) An improvised process of 610 isolation, purification of steviosides from Stevia rebaudiana Bertoni leaves and its biological activity. Int J Food Sci Tech 47:2554-2560.

612

613

Sabbioni C, Ferranti A, Bugamelli F, Forti GC, Raggi MA (2006) Simultaneous HPLC 614 analysis, with isocratic elution, of glycyrrhizin and glycyrrhetic acid in liquorice roots and 615 confectionery products. Phytochem Analysis 17:25-31. 
617 Sheng Z, Wang B, Zhao J, Yu W (2017) Optimization of ultrasonic-assisted extraction for 618 pinocembrin from flospopuli using response surface methodology. Int J Food Eng 13.

619

620 Sirichamorn Y, Adema F, Van Welzen P (2012) The Genera Aganope, Derris, and Paraderris 621 (Fabaceae, Millettieae) in Thailand. Syst Bot 37:404-436.

622

623 Wang L, Weller CL (2006) Recent advances in extraction of nutraceuticals from plants. Trends 624 Food Sci Tech 17:300-312.

625

626 Wang X, Wu Y, Chen G, Yue W, Liang Q, Wu Q (2013) Optimisation of ultrasound assisted 627 extraction of phenolic compounds from Sparganii rhizoma with response surface methodology. Ultrason Sonochem 20:846-854.

629

630

Whitehouse CR, Boullata J, McCauley LA (2008) The potential toxicity of artificial 631 sweeteners. AAOHN J 56:251-261.

632

633

634

Xia Y, Rivero-Huguet ME, Hughes BH, Marshall WD (2008) Isolation of the sweet components from Siraitia grosvenorii. Food Chem 107:1022-1028.

635

636 Yolmeh M, Najafi MBH, Farhoosh R (2014) Optimisation of ultrasound-assisted extraction of 637 natural pigment from annatto seeds by response surface methodology (RSM). Food Chem 638 155:319-324.

639 
640 Yoshikawa M, Morikawa T, Nakano K, Pongpiriyadacha Y, Murakami T, Matsuda H (2002)

641 Characterization of new sweet triterpene saponins from Albizia myriophylla. J Nat Prod $642 \quad 65: 1638-1642$.

643

644 Yusook K, Weeranantanapan O, Hua Y, Kumkrai P, Chudapongse N (2017) Lupinifolin from

645 Derris reticulata possesses bactericidal activity on Staphylococcus aureus by disrupting 646 bacterial cell membrane. J Nat Med 71:357-366. 


\section{Figures}

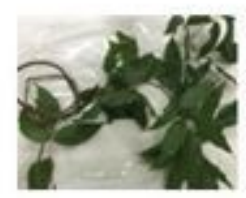

Derris reticulata stems

(Oi Sam Saun)

$$
\begin{aligned}
& \text { - Dried at } 50^{\circ} \mathrm{C} \\
& \text { - Grinded and Sieved }
\end{aligned}
$$

Oi Sam Saun stem powders

Ultrasonic-assisted extraction (UAE)

with water at $120 \mathrm{~W}$ and $37 \mathrm{~Hz}$.

$$
\begin{aligned}
& \text { - Extraction temperature }\left(40-80^{\circ} \mathrm{C}\right) \\
& \text { - Sonication time }(20-60 \mathrm{~min}) \\
& \text { - Extraction ratio }(1: 10-1: 30 \mathrm{~g} / \mathrm{mL})
\end{aligned}
$$

Oi Sam Saun UAE aqueous extracts

Determined phenolics, flavonoids and sugars by HPLC

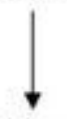

Determined yield, colour, phenolic,

flavonoid and sugar content

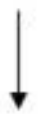

Optimized extraction condition by

Box-Behnken design (BBD) and

response surface methodology (RSM)

Optimized Oi Sam Saun UAE extract

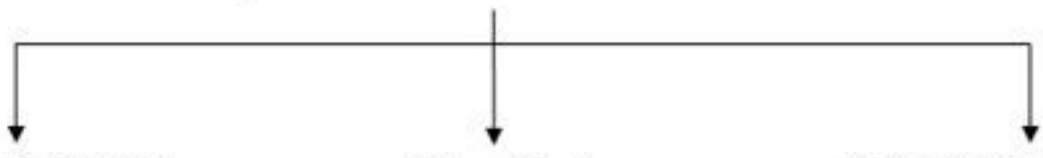

Determined sweetness

18ß-glycyrrhetinic acid by HPLC potency by sensory evaluation

\section{Figure 1}

Schematic illustration for the process of Oi Sam Saun UAE extraction and overall experiment. 
(a)
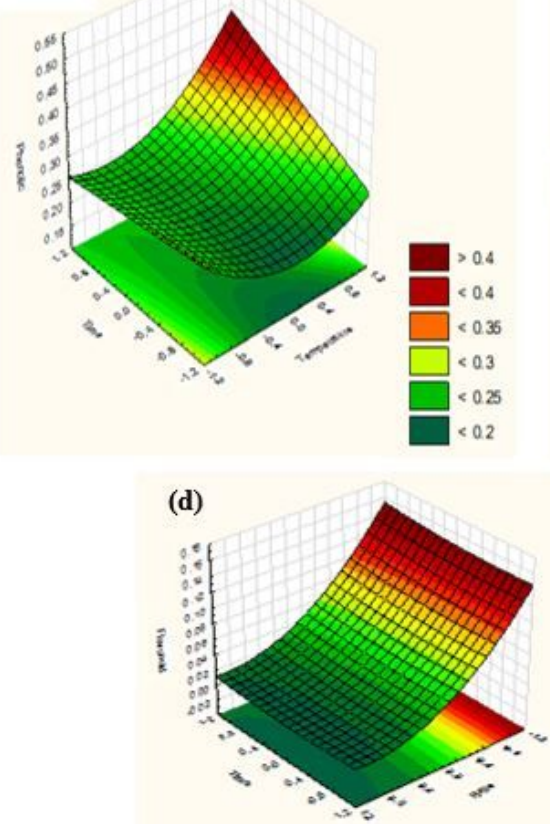

(b)

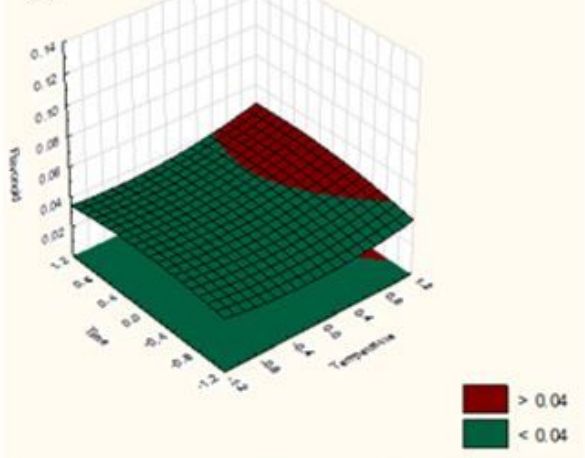

(c)

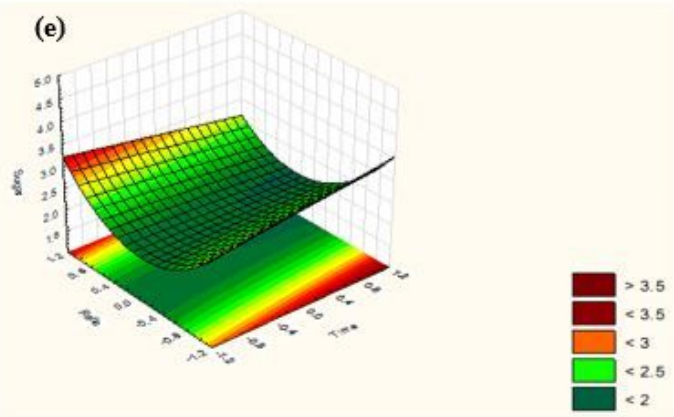

Figure 2

3D response surface graph of Oi Sam Saun of total phenolic content against time and temperature (a); total flavonoid content against time and temperature (b), temperature and ratio (c) and time and ratio (d); total sugar content against time and ratio (e).

(a)

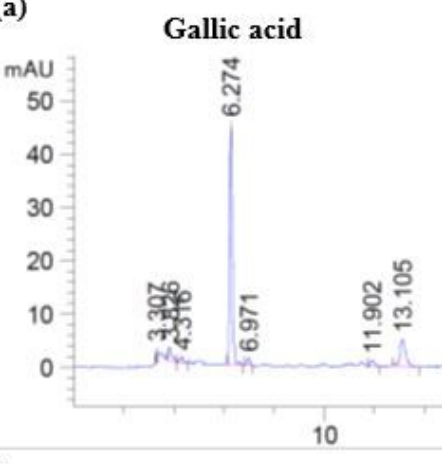

(b)

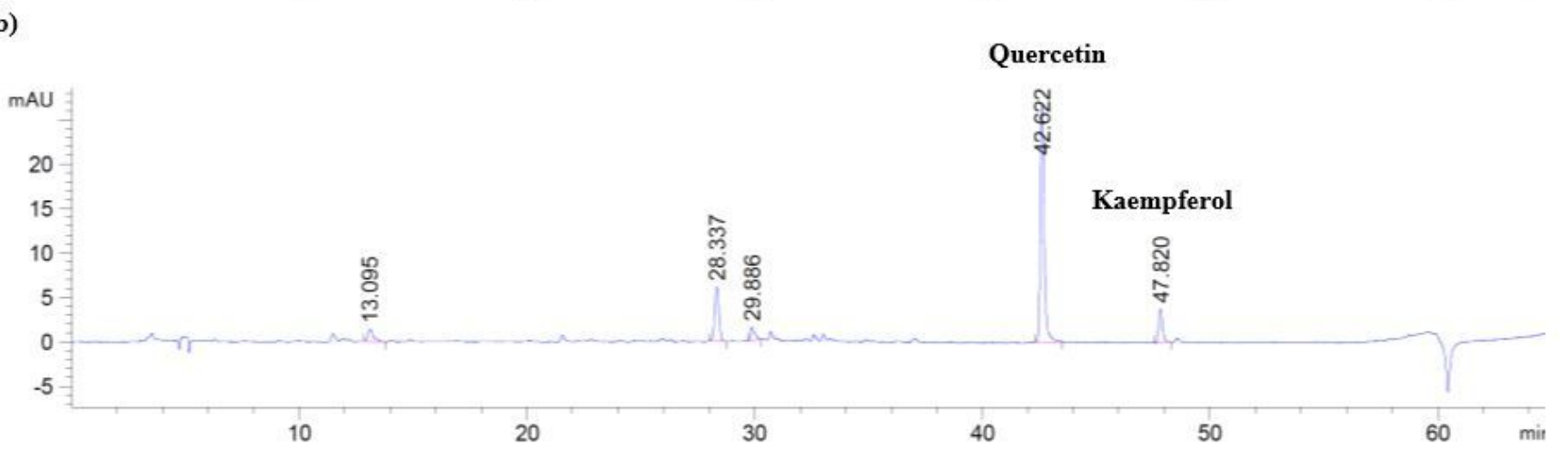

p-Coumaric acid

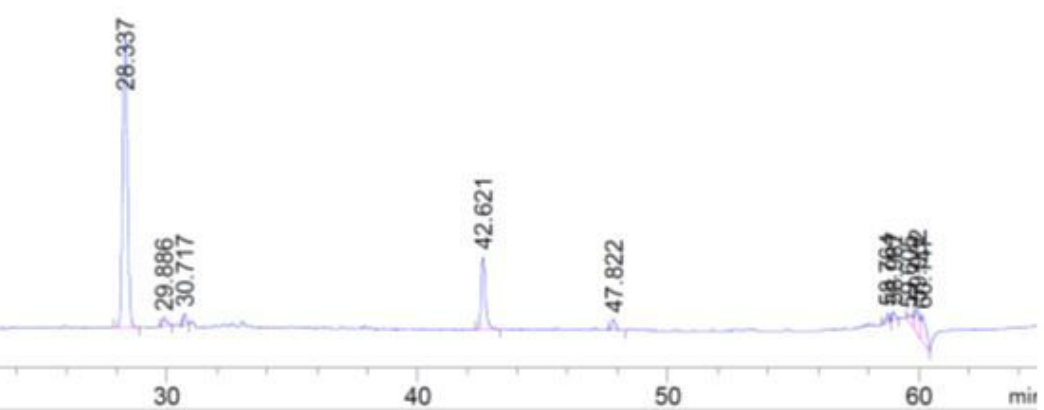

20
Quercetin

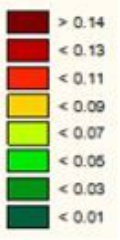


Figure 3

HPLC chromatogram showing the phenolics (a) at $280 \mathrm{~nm}$ and flavonoids (b) at $350 \mathrm{~nm}$ in the Oi Sam Saun UAE extract.

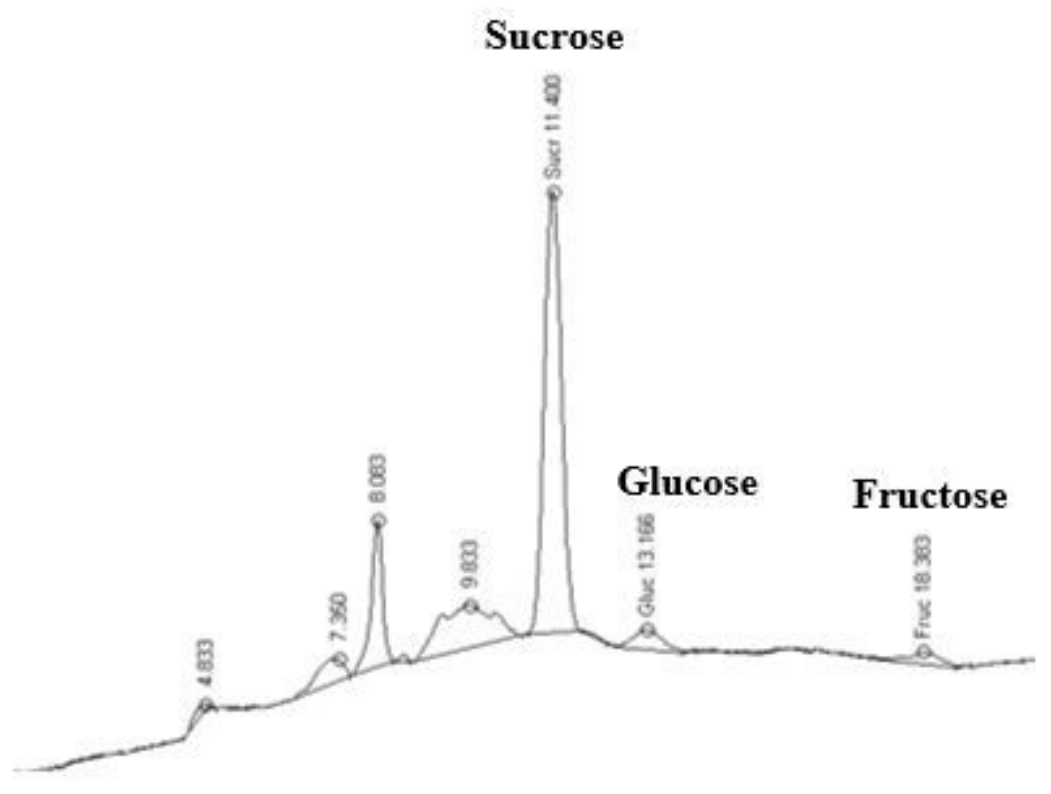

Figure 4

HPLC-ELSD (evaporating light scattering detector) chromatogram showing the sugar profile of the Oi Sam Saun UAE extract.

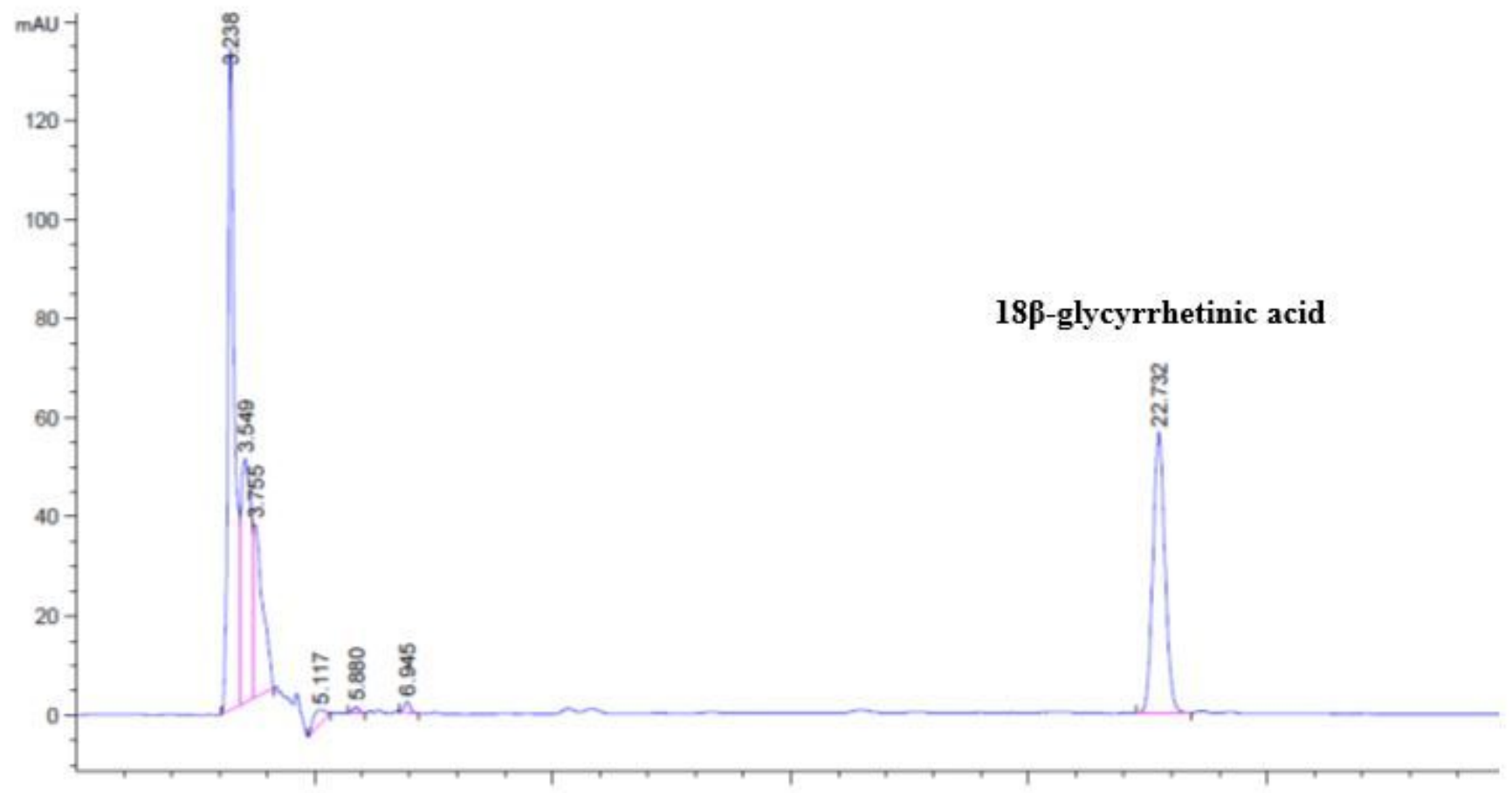


Figure 5

HPLC chromatogram showing $18 \beta$-glycyrrhetinic acid in the Oi Sam Saun UAE extract.

\section{Supplementary Files}

This is a list of supplementary files associated with this preprint. Click to download.

- Tablelegends.pdf

- GraphicalAbstracts.jpg 